\title{
A Transfer Property of Equilibrium Payoffs in Economies with Land
}

\author{
JERZY LEGUT* \\ Technical University of Wrockaw', Poland \\ Jos A. M. Potters \\ University of Nijmegen, The Netherlands \\ AND \\ STEF H. Tiss \\ University of Tilburg, The Netherlands \\ Received December 3, 1992
}

\begin{abstract}
NTU-games and TU-games are often used as tools to analyze economic models. Legut et al. (1994) investigated the properties of TU-games associated with economies with "land." In this paper we especially give attention to the NTU-games connected with these kinds of economies. The main result is that equilibrium payoffs in the NTU-model are connected to equilibrium payoff in the TU-model by $b$-transfer, a concept introduced by Shapley (1969). O 1995 Academic Press, Inc.
\end{abstract}

\section{INTRODUCTION}

We consider in this paper an exchange economy of Debreu type with only one commodity, land. This commodity is modeled by a measure space $(L, \mathscr{B}, \nu)$, wherein $\mathscr{B}$ is a $\sigma$-algebra of subsets of $L$ and $\nu: \mathscr{B} \rightarrow R_{+}$ is a non-trival finite measure on $L: 0<\nu(L)<\propto$ (cf. Berliant (1982) and Dunz (1984)).

${ }^{*}$ Financial support under KBN Grant PB 664/2/91 is gratefully acknowledged.

355

0899-8256/95 $\$ 12.00$

Copyright 1995 by Academic Press, Inc. All rights of reproduction in any form reserved. 
Utilities are given as integrals of "utility density functions" $f_{i}$ over $\nu$ measurable parcels $C \in \mathscr{B}: U_{i}(C)=\int_{C} f_{i} d \nu$.

An exchange economy with land $\mathscr{E}$ consists of

- a set of agents $N$,

- A $\mathscr{B}$-partition $\left\{A_{i}\right\}_{i \in N}$ of $L$, the initial endowments of the agents,

- a set $\left\{f_{i}\right\}_{i \in N}$ of nonnegative bounded $\nu$-measurable functions $f_{i}: L \rightarrow R_{+}$, the utility densities of the agents.

A $\mathscr{B}$-partition of $L$ is a collection subsets $A_{i} \in \mathscr{B}$ such that $\nu\left(A_{i} \cap A_{j}\right)=0$ if $i \neq j$ and $\nu\left(\cup_{i \in N} A_{i}\right)=\nu(L)$. Utilities are given by $U_{i}(C):=\int_{C} f_{i} d \nu$ for every parcel $C \in \mathscr{B}$ :

$$
\mathscr{E}=\left\{N,\left\{A_{i}, f_{i}\right\}_{i \in N}\right\}
$$

To investigate these types of economies we introduce (cf. Legut $e t$ al. (1992)) a transferable utility game $\left(N, v_{\xi}\right)$ defined by

$$
v_{\not z}(S):=\sup \left\{\sum_{i \in S} \int_{C_{i}} f_{i} d \nu \mid\left\{C_{i}\right\}_{i \in S} \text { is a } \mathscr{B} \text {-partition of } A(S)=\bigcup_{i \in S} A_{i}\right\}
$$

and a non-transferable utility game $\left(N, V_{\mathscr{E}}\right)$ with

$$
\begin{aligned}
V_{\mathscr{E}}(S) & :=\left\{x \in R^{S} \mid \text { there is a } \Re \text {-partition }\left\{C_{i}\right\}_{i \in S} \text { of } A(S) \text { with } x_{i}\right. \\
& \left.\leq \int_{C_{i}} f_{i} d \nu \text { for all } i \in S\right\} .
\end{aligned}
$$

In this paper we will prove a connection between the equilibrium payoffs of the TU-game $\left(N, v_{\mathscr{g}}\right)$ and the NTU-game $\left(N, V_{\mathscr{E}}\right)$.

The interpretation of the functions $f_{i}$ is different depending on the point of view we take. If we consider these functions from a TU-point of view, we are assuming that there is an exogeneously given medium, "money," by which utility can be transfered from one agent to another. Then the function values of $f_{i}$ have the dimension $\left[\$ / \mathrm{m}^{2}\right]$, wherein $\$$ is a unit for the exogeneously given medium "money." The utility of a parcel $C$ has the dimension [\$]. If we take an NTU-point of view, the values of $f_{i}$ have the dimension $\left[\mu_{i} / \mathrm{m}^{2}\right]$, where $\left[\mu_{i}\right]$ is the unit of subjective utility of player $i$. Then $U_{i}(C)=\int_{C} f_{i} d \nu$ has the dimension $\left[\mu_{i}\right]$. We must assume that the preferences of agent $i$ over parcels $C$ determine the utility density $f_{i}$ 
up to positive affine transformations (otherwise the integrals $\int_{C} f_{i} d \nu$ makes no sense). The utilities must be cardinally determined.

There are more differences between both interpretations of the utilities. In an NTU-setting, e.g., an economy in which one player owns all the land $\left(A_{i}=L\right.$ and $A_{j}=\varnothing$ if $\left.j \neq i\right)$ is trivial, as no trade can take place. In a TU-interpretation this economy is not trivial, as players $j \neq i$ can buy parcels from player $i$ by means of "money."

Further, the concept of competitive equilibrium is different in both contexts. In an NTU-setting a collection of $\mathscr{B}$-subsets $X=\left\{X_{i}\right\}_{i \in N}$ is a competitive equilibrium if there is a (price) density $g: L \rightarrow R_{+}$such that

(i) $\left\{X_{i}\right\}_{i \in N}$ is a partition (the market clears),

(ii) $\int_{X_{i}} g d \nu \leq \int_{A_{i}} g d \nu$ for all $i \in N$ (budget constraint), dition)

(iii) if $\int_{C} f_{i} d \nu>\int_{X_{i}} f_{i} d \nu$, then $\int_{C} g d \nu>\int_{A_{i}} g d \nu$ (maximality con-

The NTU-equilibrium payoff associated with the competitive equilibrium $\left\{X_{i}\right\}_{i \in N}$ is the $N$-vector $x$ with coordinates $x_{i}:=\int_{X_{i}} f_{i} d \nu$ for all agents $i \in N$.

In a TU-setting condition (i) stays and condition (ii) disappears: there is no budget constraint, as "money" can be used to balance any difference between selling and purchasing. Condition (iii) becomes mality)

(iii) $\int_{X_{i}}\left(f_{i}-g\right) d \nu \geq \int_{C}\left(f_{i}-g\right) d \nu$ for every parcel $C \in \mathscr{B}$ (maxi-

The TU-equilibrium payoff associated with $\left\{X_{i}\right\}_{i \in N}$ has the coordinates $x_{i}:=\int_{X_{i}}\left(f_{i}-g\right) d \nu+\int_{A_{i}} g d \nu$.

Note that in condition (iii) only integrals of the same densities are compared with each other. In (iii)' the functions $f_{i}$ and $g$ are subtracted and must therefore have the same dimension $\left[\$ / \mathrm{m}^{2}\right]$. By the way, the dimension of $g$ in the NTU-setting can be $\left[\cdot / \mathrm{m}^{2}\right]$, where "." stands for a numeral or any other unit of measurement.

In this paper we are going to compare the TU- and NTU-equilibrium payoffs. We shall prove that these equilibrium payoff are connected by "b-transfer."

First we recall the definition of the $b$-transfer of an NTU-game. Let $(N, V)$ be an NTU-game and $b \in R_{+}^{N}$. The $b$-transfer of the game $(N, V)$ is the TU-game $t_{b}(V)$ with values

$$
t_{b}(V)(S)=\sup \{\langle b, x\rangle \mid x \in V(S)\} .
$$

The set $\{\langle b, x\rangle \mid x \in V(S)\}$ is not always bounded for every coalition, but 
if it is, the $b$-transfer can be defined. In Shapley (1969) the $b$-transfer of a game is used to define the Shapley value for NTU-games. A point $x \in$ $V(N)$ is a point of the Shapley NTU-value if there is a transfer vector $b \in R_{+}^{N}$ such that the $b$-transfer $t_{b}(V)$ exists and $\left(b_{i} x_{i}\right)_{i \in N}$ is the Shapley value of the TU-game $\left(N, t_{b}(V)\right)$.

Introducing a transfer vector $b \in R_{+}^{N}$ is the same as introducing an exchange rate between the utilities of different players. If $\left(x_{1}, \ldots, x_{n}\right)$ is a point of $V(N)$, the vector $b \star x:=\left(b_{i} x_{i}\right)_{i \in N}$ is meant as a TU-payoff vector of which the coordinates are compared and added. So the dimension of the coordinate $b_{i}$ must be $\left[\cdot / \mu_{i}\right]$ or $\left[\$ / \mu_{i}\right]$.

In this paper we prove that, under mild conditions on $\mathscr{E}$, a vector $x \in$ $V_{\varepsilon}(N)$ is an NTU-equilibrium payoff if and only if there is a nonnegative transfer vector $b$ such that $b \star x$ is a TU-equilibrium payoff of $t_{b}\left(V_{q}\right)$. Furthermore, $t_{b}\left(V_{\varepsilon}\right)$ is the TU-game associated with the economy $b \star \mathscr{E}$, the economy with land, wherein each utility density $f_{i}$ is multiplied with $b_{i}$.

\section{Main Results}

As before we start with a measurable space $(L, \mathscr{B}, \nu)$, wherein $L$ is a nonempty set, $\mathscr{B}$ is a $\sigma$-algebra of subsets of $L$, and $\nu: \mathscr{B} \rightarrow R_{+}$is a finite non-trivial measure.

An economy with land consists of a finite set of agents $N$, and for each agent $i \in N$, a $\nu$-measurable initial endowment $A_{i} \subset L$ and a nonnegative (essentially) bounded $\nu$-measurable utility density $f_{i}$ on $L$. The integral $U_{i}(C):=\int_{C} f_{i} d \nu$ gives the appreciation of agent $i$ for the measurable set $C$. We assume that the following conditions hold:

(a) The measure $\nu$ is non-atomic; i.e., for every set $E \in \mathscr{B}$ with $\nu(E)>0$ there is a subset $F \subset E$ with $F \in \mathscr{B}$ and $0<\nu(F)<\nu(E)$.

(b) The utilities $f_{i}$ are almost everywhere positive: $\nu\left(\left\{x \in L \mid f_{i}(x)=0\right\}\right)=0$ for all agents $i \in N$.

(c) The economy $\mathscr{E}$ is non-trivial; i.e., at least two agents have an initial endowment of positive $\nu$-measure. An agent $i$ with $\nu\left(A_{i}\right)=0$ is called a dummy.

Let $\left\{X_{i}\right\}_{i \in N}$ be a $\overparen{B}$-partition of $L$. A coalition $S$ can improve upon $\left\{X_{i}\right\}_{i \in N}$ if there is a $\mathscr{B}$-partition $\left\{Y_{i}\right\}_{i \in S}$ of $A(S):=\cup_{i \in S} A_{i}$ such that $\int_{Y_{i}} f_{i} d \nu \geq$ $\int_{X_{i}} f_{i} d \nu$ for all agents $i \in S$ and there is at least one strict inequality. A coalition $S$ can strictly improve upon $\left\{X_{i}\right\}_{i \in N}$ if there is a $\mathscr{B}$-partition $\left\{Y_{i}\right\}_{i \in S}$ of $A(S)$ such that $\int_{Y_{i}} f_{i} d \nu>\int_{X_{i}} f_{i} d \nu$ for all agents $i \in S$. A $\Re$ partition $\left\{X_{i}\right\}_{i \in N}$ of $L$ is Pareto optimal if the coalition $N$ has no strict improvement upon $\left\{X_{i}\right\}_{i \in N}$. A $\mathscr{B}$-partition $\left\{X_{i}\right\}_{i \in N}$ of $L$ is strictly Pareto 
optimal if the coalition $N$ has no improvement upon $\left\{X_{i}\right\}_{i \in N}$. A $\mathscr{B}$-partition $\left\{X_{i}\right\}_{i \in N}$ of $L$ is a core allocation if no coalition $S$ has a strict improvement upon $\left\{X_{i}\right\}_{i \in N}$. It is a strict core allocation if no coalition $S$ has an improvement.

The following lemma states that in economies satisfying conditions (a) and (b) Pareto optimality is the same as strict Pareto optimality and the core is the same as the strict core.

LEMMA 1. If $\mathscr{E}$ is an economy with land satisfying the conditions (a) and $(b)$, then every improvement upon $\left\{X_{i}\right\}_{i \in N}$ can be changed into a strict improvement.

Proof. Let $\left\{Y_{i}\right\}_{i \in S}$ be an improvement of coalition $S$ upon $\left\{X_{i}\right\}_{i \in N}$. Then $\int_{Y_{i}} f_{i} d \nu \geq \int_{X_{i}} f_{i} d \nu$ for all agents $i \in S$ and there is a strict inequality for $i_{0} \in S$.

By non-atomicity of $\nu$ there is a subset $E \subset Y_{i_{0}}$ with positive $\nu$-measure and $\int_{E} f_{i_{0}} d \nu<\int_{Y_{i_{0}}} f_{i_{0}} d \nu-\int_{X_{i_{1}}} f_{i_{0}} d \nu$. By non-atomicity we can divide $E$ into $s-1$ parcels $E_{i}, i \in S \backslash\left\{i_{0}\right\}$ with positive $\nu$-measure. By the positivity of $f_{i}$ (condition (b)) we have $\int_{E_{i}} f_{i} d \nu>0$. Then $Z_{i_{0}}:=Y_{i_{0}} \backslash E$ together with $Z_{i}:=Y_{i} \cup E_{i}$ for $i \in S, i \neq i_{0}$ is a strict improvement of $S$.

In the proof we used the theorem of Lyapounov (1940) for the onedimensional case, saying that if $(L, \mathscr{B}, \nu)$ is a non-atomic finite measure space, $E \in \mathscr{B}$ is a $\nu$-measurable set, and $f$ is an essentially bounded nonnegative $\nu$-measurable function, then for every real number $\beta$ with $0 \leq \beta \leq \alpha:=\int_{E} f d \nu$ there is a $\nu$-measurable set $F \subset E$ with $\int_{F} f d \nu=\beta$.

It is the goal of this paper to characterize equilibrium payoffs by $b$ transfer. In the following proposition we characterize, as a first step, the Pareto optimal allocations by $b$-transfer.

Proposition 2. A R B-partition $\left\{X_{i}\right\}_{i \in N}$ of $L$ is Pareto optimal if and only if there exists a vector $b \in R_{+}^{N}$ such that $b_{i}>0$ if $\nu\left(X_{i}\right)>0$ and $X_{i} \subset^{\prime}\left\{x \in L \mid b_{i} f_{i}(x)=\max _{j \in N} b_{j} f_{j}(x)\right\}$. The notation $E \subset^{\prime} F$ means $\nu(E \backslash F)=0$.

Proof. If $b$ is as in the proposition and $\left\{Y_{i}\right\}_{i \in N}$ is a strict improvement upon $\left\{X_{i}\right\}_{i \in N}$, then

$$
\sum_{i \in N} b_{i} \int_{X_{i}} f_{i} d \nu<\sum_{i \in N} b_{i} \int_{Y_{i}} f_{i} d \nu \leq \sum_{i \in N} \int_{Y_{i}} \max _{j \in N} b_{j} f_{j} d \nu=\int_{L} \max _{j \in N} b_{j} f_{j} d \nu .
$$

Since for (almost) all points $x \in X_{i} b_{i} f_{i}(x)=\max _{j \in N} b_{j} f_{j}(x)$, we find $\sum_{i \in N} \int_{X_{i}} b_{i} f_{i} d \nu=\int_{L} \bar{f}_{b} d \nu$, where $\bar{f}_{b}=\max _{j \in N} b_{j} f_{j}$. Therefore, there is no strict improvement upon $\left\{X_{i}\right\}_{i \in N}$ and $\left\{X_{i}\right\}_{i \in N}$ is Pareto optimal. 
Conversely, let $\left\{X_{i}\right\}_{i \in N}$ be a Pareto optimal allocation. For agents $j$ with $\nu\left(X_{j}\right)=0$, we define $b_{j}=0$. Let $A$ be the set of agents $i$ whose parcel $X_{i}$ has positive $\nu$-measure. For $i, j \in A, i \neq j$, we define a real number $a_{i j}$ by

$$
\begin{aligned}
a_{i j} & :=\inf \operatorname{ess}_{X_{i}} f_{i} / f_{j} \\
& :=\sup \left\{t \in R \mid \text { the set }\left\{x \in X_{i} \mid f_{i} / f_{j}(x) \leq t\right\} \text { has } \nu \text {-measure zero }\right\} .
\end{aligned}
$$

Further we define $a_{i i}=1$ for all $i \in A$.

Claim 1. For every pair $(i, j), i, j \in A$, we have $a_{i j}<\infty$.

If $a_{i j}=x$ then the sequence of $\nu$-measurable sets $\left\{x \in X_{i} \mid f_{i} / f_{j}(x) \leq 2^{n}\right\}$ has $\nu$-measure zero. Then also the set $X_{i} \backslash f_{j}^{-1}(0)$ has $\nu$-measure zero. By property (b) we find $\nu\left(X_{i}\right)=0$.

Claim 2. If $\sigma$ is a permutation of $A$, then $\prod_{i \in A} a_{i, \sigma(i)} \geq 1$.

It is sufficient to prove that $\Pi_{i \in C} a_{i, \sigma(i)} \geq 1$ for every cycle $C$ of the permutation $\sigma$ of length $\geq 2$. Without loss of generality we assume that $C=\{1,2, \ldots, p\}$ and $\sigma(i)=i+1$ for $i=1, \ldots, p-1$ and $\sigma(p)=1$. Suppose that $\prod_{i \in C} a_{i, \sigma(i)}<1$. Choose $\bar{a}_{i, \sigma(i)}>a_{i, \sigma(i)}$ such that $\Pi_{i \in C} \bar{a}_{i, \sigma(i)}=1$. From the definition of $a_{i j}$ we infer that the sets $E_{i}:=\left\{x \in X_{i} \mid f_{i} / f_{\sigma(i)}(x)<\bar{a}_{i, \sigma(i)}\right\}$ have a positive $\nu$-measure for all $i \in C$. Let $\varepsilon>0$ be a positive number such that $\Pi_{j=1}^{i} \bar{a}_{j, \sigma(j)} \int_{E_{i}} f_{i} d \nu \geq \varepsilon$ for $i=$ $1, \ldots, p$. As $\nu$ is atomless and $f_{i}$ is positive almost everywhere, there are subsets $F_{i} \subset E_{i}$ with $\int_{F_{i}} f_{i} d \nu=\left(\Pi_{j<i} \bar{a}_{j, \sigma(j)}\right)^{-1} \varepsilon$ for all $i \in C$. The integral $\int_{F_{i}} f_{\sigma(i)} d \nu>\bar{a}_{i, \sigma(i)}^{-1} \int_{F_{i}} f_{i} d \nu=\int_{F_{\sigma(i)}} f_{\sigma(i)} d \nu$ for all $i \in C$. If we take $Y_{i}=$ $\left(X_{i} \backslash F_{i}\right) \cup F_{\sigma^{-1}(i)}$ for $i \in C$ and $Y_{j}=X_{j}$ for $j \notin C$ we have an improvement upon $\left\{X_{i}\right\}_{i \in N}$ and $\left\{X_{i}\right\}_{i \in N}$ is not (strictly) Pareto optimal.

Claim 3. There is a positive vector $b \in R_{++}^{A}$ such that $b_{j} / b_{i} \leq a_{i j}$ for all $i$ and $j \in A$.

Take $x \in R_{++}^{A}$ arbitrarily and define the mapping $\varphi: R_{++}^{A} \rightarrow R^{A}$ by $\varphi(x)_{i}:=\min _{j \in A} x_{j} a_{j i}$. As $a_{i j}>0$ for all $i, j \in A$ we have $\varphi(x)>0$ and as $a_{i i}=1$ we have $\varphi(x) \leq x$. Repeating the mapping $\varphi$ we find a weakly decreasing sequence

$$
x \geq \varphi(x) \geq \varphi^{2}(x) \geq \cdots \geq \varphi^{k}(x) \geq \cdots .
$$

On the other side, if $k>a:=|A|$, we find for each $i \in A$

$$
\varphi^{k}(x)_{i}=x_{t(k)} \cdot a_{t(k), t(k-1)} \cdots a_{t(2),(1)} \cdot a_{t(1), t(0)}
$$


for some sequence $t(0)=i, t(1), \ldots, t(k) \in A$. As $k>a$ the sequence $t(0), t(1), \ldots, t(k)$ contains repetitions and therefore by Claim 2 we have $\varphi^{k}(x)_{i} \geq \varphi^{\prime}(x)_{i}$ for some $l=l(i)<k$. Therefore the sequence $\varphi^{k}(x)$ is constant for $k \geq a$. We find a vector $b:=\varphi^{a}(x)>0$ with $b_{i}=\min _{j \in A} b_{j} a_{j i}$ for all $i \in A$.

Now we can finish the proof as follows. For almost all points of $X_{i}$, $i \in A$, we have $f_{i} / f_{j} \geq a_{i j} \geq b_{j} / b_{i}$. Then, for agents $i \in A, b_{i} f_{i}(x)=\max _{j \in A}$ $b_{j} f_{j}(x)$ for almost every point of $X_{i}$. We may replace $\max _{j \in A}$ by $\max _{j \in N}$, as $b_{j}=0$ when $j \notin A$. For $i \notin A$ the equality $b_{i} f_{i}=\max _{j \in N} b_{j} f_{j}$ almost everywhere on $X_{i}$ is an empty condition.

If $b \in R_{+}^{N}$ we introduce the notation

$$
\bar{f}_{b}^{S}=\bigvee_{i \in S} b_{i} f_{i}, \quad \bar{f}_{b}:=\bar{f}_{b}^{N}, \quad \underline{f}_{b}:=\bigwedge_{i \in N} \bar{f}_{b}^{N \backslash i},
$$

where $\bigwedge_{i \in S} b_{i} f_{i}$ and $\bigvee_{i \in S} b_{i} f_{i}$ denote the pointwise minimum and maximum of the functions $b_{i} f_{i},(i \in S)$. The function $\underline{f}_{b}$ gives in every point the second highest value.

If $\mathscr{E}=\left\{N,\left\{A_{i}, f_{i}\right\}_{i \in N}\right\}$ is an economy with land, $b \star \mathscr{E}$ is the economy with the same set of agents and initial endowments as in $\mathscr{E}$ but with utility densities $\left\{b_{i} f_{i}\right\}_{i \in N}$ instead of $\left\{f_{i}\right\}_{i \in N}$. The economy $\overline{\mathscr{E}}$ has the functions $f_{i} \vee f$ as utility densities.

It is typical for economies with land that the trades necessary to reach a reallocation $\left\{X_{i}\right\}_{i \in N}$ can be reconstructed from the reallocation $\left\{X_{i}\right\}_{i \in N}$. The parcels $A_{i} \cap X_{j}$ are sold by agent $i$ to agent $j$ and the payments (under a price regime $g$ ) are $\int_{A_{i} \cap X_{j}} g d \nu$. Therefore, it is not surprising that we need, in the proof of the main theorem, a theorem from the theory of flows. In fact we use the following theorem of Hoffman (1960).

Proposition 3 (Hoffman (1960)). If $N$ is a finite set and $L$ and $C$ are $N \times N$-matrices with $L \leq C$, then the following statements are equivalent:

(i) There is an $N \times N$-matrix $Y$ with $L \leq Y \leq C$ and $\sum_{j \neq i} Y_{i j}=$ $\sum_{j \neq i} Y_{j i}$ for all $i \in N$.

(ii) For all subsets $S \subset N$ we have $\sum_{i \in S} \sum_{j \in N \backslash S} L_{i j} \leq \sum_{i \in S} \sum_{j \in N \backslash S} C_{j i}$.

In the application of this proposition $L_{i j}$ will be $\int_{A_{i} \cap X_{j}} f_{b} d \nu$ and $C_{i j}$ will be $\int_{A_{i} \cap X_{j}} \bar{f}_{b} d \nu$. The matrix coefficients $Y_{i j}$ are the integrals $\int_{A_{i} \cap X_{j}} g d \nu$. If $f_{b} \leq g \leq \bar{f}_{b}$ the first condition of (i) is satisfied. The second part of (i) says that $\int_{A_{i} \cap X_{N i}} g d \nu=\int_{A_{N i} \cap X_{i}} g d \nu$. If we add $\int_{A_{i} \cap X_{i}} g d \nu$ we find the budget equality $\int_{A_{i}} g d \nu=\int_{X_{i}} g d \nu$.

After these preparations we formulate the rather technical first version of the main theorem: 
Proposıtion 4. A $\mathscr{B}$-partition $\left\{X_{i}\right\}_{i \in N}$ of $L$ is an NTU-competitive equilibrium in the non-trivial economy $\mathscr{E}$ if and only if there is a vector $b \in R_{+}^{N}$ with $b \neq 0$ such that

(i) $X_{i} \subset^{\prime}\left\{x \in L \mid b_{i} f_{i}(x)=\bar{f}_{b}(x)\right\}$,

(ii) $\sum_{i \in S} \int_{X_{i}} \bar{f}_{b} d \nu \geq \sum_{i \in S} \int_{A_{i}}\left(\bar{f}_{b}^{S} \vee \underline{f}_{b}\right) d \nu$.

Moreover, $b_{i}>0$ if $\nu\left(A_{i}\right)>0$.

Proof. Let $\left\{X_{i}\right\}_{i \in N}$ be an NTU-competitive equilibrium with price density $g$. Let $b_{i}$ be the infimum of the real numbers $t$ such that the set $\{x \in$ $\left.X_{i} \mid g(x) \geq t f_{i}(x)\right\}$ has $\nu$-measure zero: sup ess $x_{i} g / f_{i}$. Note that $b_{i}=0$ if $\nu\left(X_{i}\right)=0$. Let $c_{i}:=\inf \operatorname{ess}_{L \backslash X_{i}} g / f_{i}$, i.e., the supremum of the numbers $t$ with the property that the set $\left\{x \in L \backslash X_{i} \mid g(x) \leq t f_{i}(x)\right\}$ has $\nu$-measure zero.

Claim 1. $0 \leq b_{i} \leq c_{i} \leq \infty$ for all agents $i \in N$.

Suppose that $c_{i}<b_{i}$ for some agent $i \in N$. Take $\bar{b}_{i}$ and $\bar{c}_{i}$ such that $c_{i}<\bar{c}_{i}<\bar{b}_{i}<b_{i}$. The sets $Y:=\left\{x \in X_{i} \mid g(x) \geq \bar{b}_{i} f_{i}(x)\right\}$ and $Z:=\{x \in$ $\left.L \backslash X_{i} \mid g(x) \leq \bar{c}_{i} f_{i}(x)\right\}$ have positive $\nu$-measure. There is a measurable set $E \subset Y$ with $0<\int_{E} f_{i} d \nu<\int_{Z} f_{i} d \nu$ because of the non-atomicity of $\nu$ and $f_{i}>0$ almost everywhere in $X_{i}$. From the same property we infer that there is a measurable set $F \subset Z$ with $\int_{E} f_{i} d \nu<\int_{F} f_{i} d \nu \leq \bar{b}_{i} / \bar{c}_{i} \int_{E} f_{i} d \nu$. If we replace $X_{i}$ by $\bar{X}_{i}=\left(X_{i} \backslash E\right) \cup F$ we have $\int_{\bar{x}_{i}} f_{i} d \nu>\int_{X_{i}} f_{i} d \nu$ and

$$
\int_{E} g d \nu \geq \int_{E} \bar{b}_{i} f_{i} d \nu>\bar{c}_{i} \int_{F} f_{i} d \nu \geq \int_{F} g d \nu
$$

Then $g$ is not an equilibrium price for the competitive equilibrium $\left\{X_{i}\right\}_{i \in N}$. This finishes the proof of the claim.

Claim 2. If $b_{i}=0$, then $\nu\left(A_{i}\right)=0$; if $b_{i}=\infty$, then $\nu\left(A_{j}\right)=0$ for every $j \neq i$.

If $b_{i}=\mathbf{0}$ for some player $i \in N$, then $g(x)=\mathbf{0}$ for almost every point of $X_{i}$. If $\nu\left(X_{i}\right)>0$, then every player $j \neq i$ has $f_{j}>0$ almost everywhere on $X_{i}$. This means that agent $j$ can obtain $X_{i}$ costless and his appreciation for $X_{i}$ is positive. Then $\left(\left\{X_{i}\right\}, g\right)$ is not a price equilibrium. Therefore, $\nu\left(X_{i}\right)=0$. If $\nu\left(A_{i}\right)>0$ (agent $i$ is not a dummy), we have $\int_{X_{i}} f_{i} d \nu=0<$ $\int_{A_{i}} f_{i} d \nu$ and $\left(\left\{X_{i}\right\}_{i \in N}, g\right)$ is not a price equilibrium.

If $b_{i}=\infty$, we also have $c_{i}=\infty$. For every number $t>0$ we have $t f_{i}(x) \leq g(x)$ for almost every point of $L \backslash X_{j}$. As $g$ is essentially bounded, we find $f_{i}=0$ almost everywhere on $L \backslash X_{i}$. This means that $\nu\left(L \backslash X_{i}\right)=0$ or $\nu\left(X_{j}\right)=0$ for every agent $j \neq i$. As before we conclude that $\nu\left(A_{j}\right)=0$ for all agents $j \neq i$. 
In Claims 1 and 2 we proved that $0 \leq b_{i}<\infty$ for all agents $i \in N$ if the economy $\mathscr{E}$ is not trivial. Further, $b_{i}>0$ if agent $i$ is not a dummy.

From the definition of $b_{i}$ we infer that, if $i$ is not a dummy, $b_{i} f_{i} \geq g$ almost everywhere on $X_{i}$ and from the definition of $c_{i}$ and Claim 1 we find $b_{j} f_{j} \leq c_{j} f_{j} \leq g$ almost everywhere on $X_{i}$ for agents $j \neq i$. This means that $b_{i} f_{i}=\bar{f}_{b}$ almost everywhere on $X_{i}$ and $f_{b} \leq g \leq \bar{f}_{b}$ almost everywhere on $L$.

As $\int_{X_{i}} g d \nu=\int_{A_{i}} g d \nu$ for all agents $i \in N$ (budget constraint) the matrix $Y_{i j}=\int_{A_{i} \cap X_{j}} g d \nu$ is a circulation flow with lower capacity $L_{i j}=\int_{A_{i} \cap X_{j}} f_{b}$ $d \nu$ and upper capacity $C_{i j}=\int_{A_{i} \cap X_{j}} \bar{f}_{b} d \nu$. By the theorem of Hoffman we find $\int_{A(S) \cap X(N \backslash S)} \underline{f}_{b} d \nu \leq \int_{A(N \backslash S) \cap X(S)} \bar{f}_{b} d \nu$ for all $S \subset N$. Adding to both sides $\int_{A(S) \cap X(N \backslash S)} \bar{f}_{b} d \nu$, we find $\int_{X(S)} \bar{f}_{b} d \nu \geq \int_{A(S)}\left(\bar{f}_{b}^{S} \vee \underline{f}_{b}\right) d \nu$ for all $S \subset N$, as almost everywhere on $X(N \backslash S)$ the function $\bar{f}_{b}^{S} \leq \bar{f}_{b}$ and on $X(S)$ the function $\bar{f}_{b}^{S}=\bar{f}_{b} \geq f_{b}$.

Conversely, if there is a vector $b$ satisfying the conditions of the theorem, we prove that there is a bounded $\nu$-measurable function $g$ such that

$$
\underline{f}_{b} \leq g \leq \bar{f}_{b} \quad \text { and } \quad \int_{X_{i}} g d \nu=\int_{A_{i}} g d \nu
$$

If we define $L_{i j}$ and $C_{i j}$ as before, condition (ii) of Proposition 4 implies that the condition in the theorem of Hoffman is satisfied and there is a circulation flow $Y=\left(Y_{i j}\right)$ with $L_{i j} \leq Y_{i j} \leq C_{i j}$ for all $i \neq j$. If $Y_{i j}=$ $\lambda_{i j} L_{i j}+\left(1-\lambda_{i j}\right) C_{i j}$ with $\lambda_{i j} \in[0,1]$, we define $g_{\mid A_{i} \cap X_{j}}$ by $\left(\lambda_{i j} f_{b}+\right.$ $\left.\left(1-\lambda_{i j}\right) \bar{f}_{b}\right)_{\mid A_{i} \cap X_{j}}$. Then $\underline{f}_{b} \leq g \leq \bar{f}_{b}$ and $\int_{A_{i} \cap X_{j}} g d \nu=Y_{i j}$. On $A_{i} \cap \bar{X}_{i}$ the function $g$ can be chosen arbitrarily between $f_{b}$ and $\bar{f}_{b}$.

Finally, we prove that the price density $g$ satisfies the maximality condition (iii). Let $i$ be any agent and let $C \in \mathscr{B}$ be a parcel of land with $\int_{C} f_{i} d \nu>\int_{X_{i}} f_{i} d \nu$. Then $C \backslash X_{i}$ has a positive measure and $b_{i} f_{i} \leq f_{b}$ almost everywhere on $C \backslash X_{i}$. Further, $b_{i} f_{i}=\bar{f}_{b}$ almost everywhere on $\bar{X}_{i}$.

Then we have, if $b_{i}>0$,

$$
\begin{aligned}
\int_{C \backslash X_{i}} g d \nu-\int_{X_{i} \backslash C} g d \nu & \geq \int_{C \backslash X_{i}} f_{b} d \nu-\int_{X_{i} \backslash C} \bar{f}_{b} d \nu \\
& \geq \int_{C \backslash X_{i}} b_{i} f_{i} d \nu-\int_{X_{i} \backslash C} b_{i} f_{i} d \nu>0
\end{aligned}
$$

If $b_{i}=0$, then $\nu\left(X_{i}\right)=0$ and $\int_{C \backslash X_{i}} g d \nu \geq \int_{C \backslash X_{i}} f_{b} d \nu>0$ if $f_{b}>0$ almost everywhere on $C$. This is clearly true if at least two players have a positive coordinate in $b$. If the economy is not trivial, the price of $C$ is larger than 
the price of $X_{i}$ (and of $A_{i}$ ). The function $g$ is a price density to the $\mathscr{B}$ partition $\left\{X_{i}\right\}_{i \in N}$.

Before we come to the final version of the main theorem we have to recall some results of Legut et al. (1994).

( $\alpha$ ) In Legut et al. (1994) it is proved that a $\mathscr{B}$-partition $\left\{X_{i}\right\}_{i \in N}$ is a TU-competitive equilibrium if and only if, for all agents $i \in N, X_{i} \subset^{\prime}\{x \in$ $\left.L \mid f_{i}(x)=\bar{f}(x)\right\}$. As $\left\{X_{i}\right\}_{i \in N}$ is a $\mathscr{B}$-partition, the set $Y_{i}:=\left\{x \in L \mid f_{i}(x)=\right.$ $\bar{f}(x)>f(x)\} \subset^{\prime} X_{i}$. The equilibrium price can be any function between $f$ and $\bar{f}$. Condition (i) of Proposition 4 states that $\left\{X_{i}\right\}_{i \in N}$ is a TU-competitive equilibrium in the economy $b \star \mathscr{E}$.

$(\beta)$ For an economy with land $\mathscr{E}$ the TU-game $v_{\mathscr{E}}$ as defined in the Introduction has the following values:

$$
v_{\dot{\varepsilon}}(S)=\int_{A(S)} \bar{f}^{S} d \nu
$$

Rewriting condition (ii) we find for the right hand side

$$
\sum_{i \in S} \int_{A_{i}}\left(\bar{f}_{b}^{S} \vee \underline{f}_{b}\right) d \nu=\int_{A(S)}\left(\bar{f}_{b}^{S} \vee \underline{f}_{b}\right) d \nu=v_{\overline{b+\varepsilon} \bar{\varepsilon}}(S),
$$

where $\overline{b \star \mathscr{E}}$ is the economy with the same set of agents and initial endowments as $\mathscr{E}$ but with utilities $b_{i} f_{i} \vee f_{b}, i \in N$. Condition (ii) of Proposition 4 says that $\left\{\int_{X_{i}} \bar{f}_{b} d \nu\right\}_{i \in N}$ is a core element of the TU-game $v_{\overline{b \star \varepsilon}}$.

( $\gamma$ ) Finally, for a TU-competitive equilibrium $\left\{X_{i}\right\}_{i \in N}$, the price density $g$ (arbitrarily chosen between $f$ and $f$ ) determines the TU-equilibrium payoff

$$
x_{i}:=\int_{X_{i}}\left(f_{i}-g\right) d \nu+\int_{A_{i}} g d \nu \quad \text { for all } i \in N
$$

completely. It is proved that $\left\{x_{i}\right\}_{i \in N}$ is a TU-equilibrium payoff if and only if $x \in \operatorname{Core}\left(v_{\bar{b}}\right)$. Hence, as $\bar{f}_{b}=b_{i} f_{i}$ almost everywhere on $X_{i}$, condition (ii) of Proposition 4 is equivalent with

$$
\begin{aligned}
& \left(b_{i} \int_{X_{i}} f_{i} d \nu\right)_{i \in N} \text { is a TU-equilibrium payoff with respect to } \\
& \left\{X_{i}\right\}_{i \in N} \text { in the economy } b \star \mathscr{E} .
\end{aligned}
$$

Summarizing we have 
THEOREM 5. A $\Re$-partition $\left\{X_{i}\right\}_{i \in N}$ is an NTU-competitive equilibrium of a non-trivial economy with land $\mathscr{E}=\left\{N,\left\{A_{i}, f_{i}\right\}_{i \in N}\right\}$ if and only if there is a transfer vector $b \in R_{+}^{N}$ (with $b_{i}>0$ if $\nu\left(A_{i}\right)>0$ ) such that

(i) $\left\{X_{i}\right\}_{i \in N}$ is a TU-competitive equilibrium of the economy $b \star \mathscr{E}$,

(ii) $b \star\left(\int_{X_{i}} f_{i} d \nu\right)_{i \in N}$ is a TU-equilibrium payoff with respect to $\left\{X_{i}\right\}_{i \in N}$ in the economy $b \star \mathscr{E}$.

For equilibrium payoffs we have

COROLlARY. If $f \mathscr{E}$ is a non-trivial economy with land and $x$ is an element of $V_{\varepsilon}(N)$, then $x$ is an NTU-equilibrium payoff iff there is a transfer vector $b \in R_{+}^{N}$ (with $b_{i}>0$ if $\nu\left(A_{i}\right)>0$ ) such that $b \star x$ is a TU-equilibrium payoff in the economy $b \star \mathscr{E}$.

Proof. Let $x \in V_{\mathscr{B}}(N)$ and let $b \in R_{+}^{N}$ be a vector such that $b_{i}>0$ if $\nu\left(A_{i}\right)>0$ and $b \star x$ is a TU-equilibrium payoff of $b \star \mathscr{C}$.

As $x \in V_{\mathscr{E}}(N)$, there is $\mathscr{B}$-partition $\left\{X_{i}\right\}_{i \in N}$ such that $x_{i} \leq \int_{X_{j}} f_{i} d \nu$ for all agents $i \in N$. We prove that $\left\{X_{i}\right\}_{i \in N}$ satisfies the conditions of Theorem 5 .

If we multiply these inequalities with $b_{i}$ and sum over $i \in N$, we find

$$
\langle b, x\rangle \leq \sum_{i \in N} \int_{X_{i}} b_{i} f_{i} d \nu
$$

Since $b \star x$ is a TU-equilibrium payoff in $b \star \mathscr{E}$, we have in particular that $\langle b, x\rangle=v_{b \star \mathscr{E}}(N)=\int_{L} \bar{f}_{b} d \nu$ and therefore all inequalities in the preceding formula are equalities: $b_{i} x_{i}=\int_{X_{i}} b_{i} f_{i} d \nu=\int_{X_{i}} \bar{f}_{b} d \nu$ for all agents $i \in N$. This means that $X_{i} \subset^{\prime}\left\{x \in L \mid b_{i} f_{i}(x){ }^{\prime}=\bar{f}_{b}(x)\right\}$ and $\left\{X_{i}\right\}_{i \in N}$ is a TU-competitive equilibrium in $b \star \mathscr{E}$ by $(\alpha)$ (condition (i)). Moreover, $b \star x=b \star\left(\int_{X_{i}} f_{i} d \nu\right)_{i \in N}$ is a TU-equilibrium payoff in the economy $b \star \mathscr{E}$ (condition (ii)).

\section{Concluding Remarks}

In this final section we will answer three fundamental questions about the model we used in this paper. ${ }^{1}$

First the meaning of a TU-model and particularly of TU-equilibria can be questioned.

\footnotetext{
${ }^{1}$ We are grateful to one of the referees who raised these questions.
} 
(1) What is the meaning of $T U$-models and especially of $T U$-equilibria? Are price equilibria without budget constraint not strange to all thinking about economies?

Indeed, a TU-model needs many preassumptions to make sense. It assumes, e.g., that there is an externality like money, equally appreciated by all the agents, and that in the economy to be considered "money is not the problem" or, to say it differently, every player must have enough "outside assets" to balance the difference between his buyings and his sellings. Under these circumstances it makes sense to fix a price that "clears the market" and gives after all the agents a payoff, partially in goods (land) and partially in money, that is as high as possible. This is exactly the TU-equilibrium concept we propose. In fact, there is some tradition in modeling economic situations by TU-games (see, e.g., the house market model of Shapley and Shubik (1972) and Kaneko (1976), (1982)). Finally this paper shows that TU-equilibria are tightly connected with economically not suspect NTU-equilibria.

The second question raises the issue of how far a commodity like "land" differs from traditional commodities.

(2) What is special to the commodity "land" compared with "normal commodities"?

The main difference is the uniqueness of parcels of land. A parcel can be bought from the owner and from nobody else. This makes it possible to trace back who bought what parcel from whom after the trades are made. In an economy with normal commodities (like crude oil or milk) this cannot be done uniquely. In the paper we use this idea by comparing the trading behavior with a flow of goods and money. Loosely speaking, land can be seen as an overwhelming number of indivisible goods (the points of $L$ ) that can be traded only in some combinations (the measurable sets).

The last question is about the kind of utility functions we considered.

(3) What do the utility functions have the form they have?

Indeed, a difficult commodity like land requires a more sophisticated utility function. In our first paper on this subject (Legut et al. (1992)) we referred to the Ph.D. dissertations of Berliant (1982) and Dunz (1984), wherein attempts are made to consider more general utility functions. But even the most general utility concept we know of (utility functions only continuous with respect to an atomless measure (Dunz (1984)) cannot deal with all the intricacies of a commodity like land. Here global geometrical or topological concepts like shape of parcels, connectedness, or 1connectedness (there no enclaves owned by somebody else) play an important role. Therefore we used the simpler utility functions, as the more sophisticated ones are not doing the job better. 


\section{REFERENCES}

Berliant, M. (1982). A General Equilibrium Model of an Economy with Land. Ph.D. Thesis, University of California, Berkeley, CA.

Dunz, K. (1984). Some Results of Economies with Land. Ph.D. Thesis, University of California, Berkeley, CA.

HofFMAN, A. J. (1960). "Some Recent Applications of the Theory of Linear Inequalities to Extremal Combinatorial Analysis," Proc. Appl. Math. 10, 113-127.

Kaneko, M. (1976). "On the Core and Competitive Equilibria of a Market with Indivisible Goods," Nav. Res. Log. Quart. 23, 321-337.

Kaneko, M. (1982). "The Central Assignment Game and the Assignment Markets," J. Math. Econ. 10, 205-232.

Legut, J., Potters, J. A. M., And Tus, S. H. (1994). "Economies with Land: A Game Theoretic Approach," Games Econ. Behav. 6, 414-430.

Lyapounov, A. A. (1940). "On Completely Additive Vector Functions," Izv. Akad. Nauk. USSR, 465-478. [in Russian]

Shapley, L. S. (1969). "Utility Comparison and the Theory of Games." La Décision 2, 251-263.

Shapley, L. S., and Shubik, M. (1972). "The Assignment Game I: The Core," Int. J. Game Theory 1, 111-131. 